International Journal of Clinical Dermatology \& Research (IJCDR)

ISSN 2332-2977

\title{
The Association Between Pressure Ulcer Development And Patient Comorbidities In Varied Care Settings: A Review Of The Literature
}

Kurashige $\mathrm{Y}$

Review Article

Department of Dermatology, Hachioji Medical Center, Tokyo Medical University, 1163 Tatemachi, Hachioji-shi, Tokyo 193-0998, Japan.

\begin{abstract}
Pressure ulcers can be described as localized injury to the skin which can develop in the patients placed in various types of care setting. The comorbidities, or underlying diseases of the patients, are thought to be strong risk factors for the development of pressure ulcers. The purpose of this paper is to review the previous literature investigating the association between patient comorbidities and pressure ulcer development. The author classified the literature into five categories by the type of care setting, namely, the nursing home, hospital, perioperative, rehabilitation, and home settings, and summarized the study results for each. The author found a large dispersion in the results according to design and sample size, even within the same settings. Nonetheless, half or more of the studies found that diabetes mellitus had a close association with pressure ulcer development in all of the settings while cardiac, renal, and respiratory diseases were also found to be associated with many of the settings. The author surmises that recognition of such comorbidities could serve as an important step towards improving pressure ulcer prevention.
\end{abstract}

KeyWords: Cardiac Disease; Comorbidity; Diabetes Mellitus; Pressure Ulcer; Renal Disease; Respiratory Disease; Underlying Disease

\section{*Corresponding Author:}

Yuta Kurashige,

Department of Dermatology,

Hachioji Medical Center, Tokyo Medical University,

1163 Tatemachi, Hachioji-shi, Tokyo 193-0998, Japan.

Tel: +81.42.665.5611; Fax: +81.42.665.1796

E-mail: kurasige@tokyo-med.ac.jp

Received: January 27, 2014

Accepted: February 19, 2014

Published: February 21, 2014

Citation: Kurashige Y (2014) The Association Between Pressure Ulcer Development and Patient Comorbidities in Varied Care Settings: A Review of The Literature. Int J Clin Dermatol Res. 2(1), 4-8. doi: http:// dx.doi.org/10.19070/2332-2977-140002

Copyright: Yuta Kurashige ${ }^{\odot} 2014$. This is an open-access article distributed under the terms of the Creative Commons Attribution License, which permits unrestricted use, distribution and reproduction in any medium, provided the original author and source are credited

\section{Introduction}

A pressure ulcer (PU) is defined as "localized injury to the skin and/ or underlying tissue, usually over a bony prominence, as a result of pressure or pressure in combination with shear" [1]. In addition to such mechanical forces, however, patient comorbidity or an underlying disease may also contribute to PU development. Several clinical guidelines for PU prevention refer to comorbidity as a risk factor for PU development, citing, for example, diabetes mellitus [1-4], circulatory disease [1], spinal cord injury [2,4], hip fracture [4], cerebrovascular disease [4], etc. In addition, risk assessment scales for PU prevention such as CBO[5], Spinal Cord Injury Pressure Ulcer Scale (SCIPUS)[6], SCIPUS-A[7] and Waterlow [8] include factors related to patient comorbidities such as diabetes mellitus, and pulmonary and cardiac diseases.

PUs can develop in patients placed in any types of care setting. The aim of this paper is to review the previous English-language literature investigating the association between patient comorbidities and PU development. The author classified the literature into five categories according to the type of care setting, namely the nursing home, hospital, perioperative, rehabilitation, and home settings, and further examined the kind of PU-related comorbidity in each of these settings.

\section{Nursing Home Setting}

Nursing homes constitute representative long-term care settings for the immobile or inactive elderly population. The previous literature dealing with the association between comorbidities and PU development in nursing homes is summarized in Table 1. To the author's knowledge, seven studies have been published to date on this subject [9-15], three cohort studies [10,13,14] and four cross-sectional studies $[9,11,12,15]$, all of which utilized large databases at multiple facilities. It should be noted that six of the seven studies cited $[9,11,15]$ concluded that diabetes mellitus was associated with PU development. Moreover, in some studies, hip fracture [13], Parkinson's disease [9], and peripheral vascular disease were also listed as risk factors, while other studies showed inverse results $[9,11,12,14]$.

\section{Hospital Setting}

Inpatient hospital care ranges from acute or intensive care to chronic or geriatric care. Accordingly, hospitalized patients may present many types of comorbidities in association with PU development. As shown in Table 2, we found three cohort studies [18-20], three cross-sectional studies [16,21,22], one crosssectional/cohort study [17], and one case-control study[23], or a total of eight studies dealing with PU-related comorbidities in the hospital setting. Three of these studies[21-23] utilized a large inpatient database derived from multiple facilities. On the other hand, six [16-20] utilized relatively small samples from single hospitals including two teaching hospitals[16,19], two chronic care hospitals [17,18], and one medical ICU in a public hospital [20]. In four of the studies [17,21-23], diabetes mellitus was described 
Table 1. Nursing home setting

\begin{tabular}{|c|c|c|c|c|c|c|}
\hline Study & Study design & Participants & $\begin{array}{l}\text { Study setting and } \\
\text { database }\end{array}$ & Country & $\begin{array}{l}\text { Comorbidities in } \\
\text { associated with PU } \\
\text { development }\end{array}$ & $\begin{array}{l}\text { Comorbidities not } \\
\text { associated with PU } \\
\text { development }\end{array}$ \\
\hline Spector, 1994 [9] & Cross-sectional & 2803 & $\begin{array}{l}699 \text { nursing homes in } \\
\text { NMES IPC } 1987\end{array}$ & United States & $\begin{array}{l}\text { Diabetes mellitus, } \\
\text { Parkinson's disease }\end{array}$ & Hip fracture, stroke \\
\hline $\begin{array}{l}\text { Brandies et al, } 1994 \\
{[10]}\end{array}$ & Cohort & 4232 & $\begin{array}{l}78 \text { nursing homes belong- } \\
\text { ing to National Health } \\
\text { Corp }\end{array}$ & United States & None & Diabetes mellitus \\
\hline $\begin{array}{l}\text { Brandies et al, } 1995 \\
{[11]}\end{array}$ & Cross-sectional & 2011 & $\begin{array}{l}270 \text { nursing homes in MDS } \\
1990\end{array}$ & United States & Diabetes mellitus & $\begin{array}{l}\text { Alzheimer's disease, hip } \\
\text { fracture, peripheral vascu- } \\
\text { lar disease }\end{array}$ \\
\hline $\begin{array}{l}\text { Spector and Fortin- } \\
\text { sky, } 1998 \text { [12] }\end{array}$ & Cross-sectional & 15121 & $\begin{array}{l}843 \text { nursing homes in } \\
\text { MDS }+1994\end{array}$ & United States & Diabetes mellitus & $\begin{array}{l}\text { Parkinson's disease, hip } \\
\text { fracture, stroke }\end{array}$ \\
\hline $\begin{array}{l}\text { Berlowitz et al, } 2001 \\
{[13]}\end{array}$ & Cohort & 14607 & $\begin{array}{l}109 \text { nursing homes in MDS } \\
1997\end{array}$ & United States & $\begin{array}{l}\text { Diabetes mellitus, hip } \\
\text { fracture, peripheral } \\
\text { vascular disease }\end{array}$ & None \\
\hline $\begin{array}{l}\text { Berlowitz et al, } 2001 \\
{[14]}\end{array}$ & Cohort & 13457 & $\begin{array}{l}108 \text { nursing homes in MDS } \\
1998\end{array}$ & United States & Diabetes mellitus & $\begin{array}{l}\text { End-stage disease, hip } \\
\text { fracture, peripheral vascu- } \\
\text { lar disease }\end{array}$ \\
\hline $\begin{array}{l}\text { Casimro et al, } 2002 . \\
{[15]}\end{array}$ & Cross-sectional & 827 & 50 geriatric facilities & United Kingdom & Diabetes mellitus & None \\
\hline
\end{tabular}

NMES IPC (Institutional Population Component of the National Medical Expenditure Survey): A nationally representative sample of residents in nursing homes.

MDS+ (Minimum Data Set Plus): The expanded version of a national resident assessment instrument specifically for Multistate Nursing Home Case Mix and Quality Demonstration Project.

MDS (Minimum Data Set): A valuable tool for assessing the quality of nursing home care containing clinical information describing the health status of all nursing home residents.

Table 2. Hospital settings

\begin{tabular}{|c|c|c|c|c|c|c|}
\hline Study & Study design & Participants & $\begin{array}{l}\text { Study setting and } \\
\text { database }\end{array}$ & Country & $\begin{array}{l}\text { Comorbidities in associated } \\
\text { with PU development }\end{array}$ & $\begin{array}{l}\text { Comorbidities not associ- } \\
\text { ated with PU development }\end{array}$ \\
\hline $\begin{array}{l}\text { Allman et al, } \\
1996[16]\end{array}$ & Cross-sectional & 634 & Single teaching hospital & United States & Fracture & $\begin{array}{l}\text { Anemia, dementia, diabetes } \\
\text { mellitus, heart failure, } \\
\text { malignancy, pneumonia, } \\
\text { renal failure, spinal cord } \\
\text { injury, stroke, urinary tract } \\
\text { infection }\end{array}$ \\
\hline $\begin{array}{l}\text { Berlowitz and } \\
\text { Wilking, } 1989 \\
{[17]}\end{array}$ & $\begin{array}{l}\text { Cross-sectional } \\
\text { and cohort }\end{array}$ & 301 & $\begin{array}{l}\text { Single chronic care } \\
\text { hospital }\end{array}$ & United States & $\begin{array}{l}\text { Diabetes mellitus, cerebrovascu- } \\
\text { lar accident }\end{array}$ & $\begin{array}{l}\text { Dementia, diabetes mel- } \\
\text { litus, heart failure, fracture, } \\
\text { malignancy }\end{array}$ \\
\hline $\begin{array}{l}\text { Bianchetti et } \\
\text { al, } 1993[18]\end{array}$ & Cohort & 148 & $\begin{array}{l}\text { Single psychogeriatric } \\
\text { hospital }\end{array}$ & Italy & None & $\begin{array}{l}\text { Diabetes mellitus, heart } \\
\text { failure, peripheral vascular } \\
\text { disease, pneumonia, stroke }\end{array}$ \\
\hline $\begin{array}{l}\text { Allman et al, } \\
1995 \text { [19] }\end{array}$ & Cohort & 286 & Single teaching hospital & United States & None & $\begin{array}{l}\text { Chronic heart failure, } \\
\text { diabetes mellitus, fracture, } \\
\text { paraplegia }\end{array}$ \\
\hline $\begin{array}{l}\text { Jiricka et al, } \\
1995[20]\end{array}$ & Cohort & 85 & $\begin{array}{l}\text { Medical/ surgical } \\
\text { ICU in a single public } \\
\text { hospital }\end{array}$ & United States & None & Diabetes mellitus \\
\hline $\begin{array}{l}\text { Scott et al, } \\
2006[21]\end{array}$ & Cross-sectional & 100000 & CHARS 1987-2000 & United States & $\begin{array}{l}\text { Diabetes mellitus, spinal cord } \\
\text { injury }\end{array}$ & Injury, infection \\
\hline $\begin{array}{l}\text { Fogerty, } 2008 \\
{[22]}\end{array}$ & Cross-sectional & 94758 & NIS 2003 & United States & $\begin{array}{l}\text { Anemia, cerebrovascular } \\
\text { disease, chronic heart failure, } \\
\text { diabetes mellitus, osteomyelitis, } \\
\text { pneumonia, renal failure, respira- } \\
\text { tory failure, sepsis, urinary tract } \\
\text { infection }\end{array}$ & Not described \\
\hline $\begin{array}{l}\text { Lyder et al, } \\
2012[23]\end{array}$ & Case-control & 51842 & MPSMS 2006-2007 & United States & $\begin{array}{l}\text { Cerebrovascular disease, chronic } \\
\text { heart failure, chronic obstruc- } \\
\text { tive pulmonary disease, diabetes } \\
\text { mellitus }\end{array}$ & None \\
\hline
\end{tabular}

CHARS (Comprehensive Hospital Abstract Reporting System): The annual data from all hospital admissions in the Washington state. NIS (Nationwide Inpatient Sample): A national sample of inpatient discharge data.

MPSMS (Medical Patient Safety Monitoring System): A nationwide surveillance system within the hospitalized fee-for-service Medicare population. 
as a risk factor for PU development, although the remaining four studies denied this association [16,18-20]. Additionally, anemia [20], cerebrovascular disease[17,22,23], chronic heart failure[22,23], chronic obstructive pulmonary disease [23], fracture [16], osteomyelitis [22], pneumonia[22], renal failure[22], respiratory failure [22], sepsis [22], spinal cord injury [21], and urinary tract infection [22] were mentioned as risk factors for PU development.

\section{Perioperative Setting}

Patients who undergo a lengthy operation are potentially at high risk for PU development because repositioning may not be possible for several hours during the intraoperative and postoperative periods. The author has found 10 studies to date concerning PU-related comorbidities in the perioperative setting [24-33], including nine cohort studies [24-31,33] and one meta-analysis [32](Table 3). Eight of these studies were based on data from a

Table 3. Perioperative settings

\begin{tabular}{|c|c|c|c|c|c|c|}
\hline Study & $\begin{array}{l}\text { Study } \\
\text { design }\end{array}$ & Participants & $\begin{array}{l}\text { Study setting and } \\
\text { database }\end{array}$ & Country & $\begin{array}{l}\text { Comorbidities in } \\
\text { associated with } \\
\text { PU development }\end{array}$ & $\begin{array}{l}\text { Comorbidities not asso- } \\
\text { ciated with PU develop- } \\
\text { ment }\end{array}$ \\
\hline $\begin{array}{l}\text { Papantonio et al, } 1994 \\
{[24]}\end{array}$ & Cohort & $\begin{array}{l}136 \text { (cardiac sur- } \\
\text { gery patients) }\end{array}$ & Single teaching hospital & United States & $\begin{array}{l}\text { Diabetes mellitus, } \\
\text { respiratory disease }\end{array}$ & $\begin{array}{l}\text { Hypertension, periph- } \\
\text { eral vascular disease, renal } \\
\text { disease }\end{array}$ \\
\hline Lewicki et al, 1997 [25] & Cohort & $\begin{array}{l}337 \text { (cardiac sur- } \\
\text { gery patients) }\end{array}$ & $\begin{array}{l}\text { Single academic medical } \\
\text { center }\end{array}$ & United States & Diabetes mellitus & None \\
\hline Schultz et al, 1999 [26] & Cohort & 413 & $\begin{array}{l}\text { Single tertiary care } \\
\text { center }\end{array}$ & United States & Diabetes mellitus & None \\
\hline Pokorny et al, 2003 [27] & Cohort & $\begin{array}{l}351 \text { (cardiac sur- } \\
\text { gery patients) }\end{array}$ & Single medical center & United States & Heart failure & None \\
\hline Frankel et al, 2007 [28] & Cohort & 820 & $\begin{array}{l}\text { Surgical ICU in a single } \\
\text { teaching hospital }\end{array}$ & United States & $\begin{array}{l}\text { Diabetes mellitus, } \\
\text { spinal cord injury }\end{array}$ & None \\
\hline Haleem et al, 2008 [29] & Cohort & $\begin{array}{l}4546 \text { (hip fracture } \\
\text { surgery patients) }\end{array}$ & Single hospital & United Kingdom & Diabetes mellitus & $\begin{array}{l}\text { Malignant disease, Rheu- } \\
\text { matoid arthritis }\end{array}$ \\
\hline Lindholm et al, 2008 [30] & Cohort & $\begin{array}{l}635 \text { (hip fracture } \\
\text { surgery patients) }\end{array}$ & $\begin{array}{l}\text { Accident and Emer- } \\
\text { gency Departments in } \\
\text { six countries }\end{array}$ & $\begin{array}{l}\text { Sweden, Finland, } \\
\text { United King- } \\
\text { dom, Spain, Italy, } \\
\text { Portugal }\end{array}$ & $\begin{array}{l}\text { Cardiovascular } \\
\text { disease, diabetes } \\
\text { mellitus, pulmonary } \\
\text { disease }\end{array}$ & $\begin{array}{l}\text { Gastrointestinal disease, } \\
\text { malignancy, urological } \\
\text { disease }\end{array}$ \\
\hline $\begin{array}{l}\text { Slowikowski and Funk, } \\
2010 \text { [31] }\end{array}$ & Cohort & 369 & $\begin{array}{l}\text { Surgical ICU in a single } \\
\text { hospital }\end{array}$ & United States & Diabetes mellitus & $\begin{array}{l}\text { Renal disease, vascular } \\
\text { disease }\end{array}$ \\
\hline Liu et al, 2012 [32] & $\begin{array}{l}\text { Meta-anal- } \\
\text { ysis }\end{array}$ & 2453 & Six observational studies & $\begin{array}{l}\text { United States (5 } \\
\text { studies) and Bel- } \\
\text { gium (one study) }\end{array}$ & Diabetes mellitus & None \\
\hline O’ Brien et al, 2013 [33] & Cohort & 2695 & $\begin{array}{l}\text { Surgical ICUs in a single } \\
\text { teaching hospital }\end{array}$ & United States & $\begin{array}{l}\text { Congestive heart } \\
\text { failure, renal failure }\end{array}$ & Hypertension, liver disease \\
\hline
\end{tabular}

Table 4. Rehabilitation settings

\begin{tabular}{|c|c|c|c|c|c|c|}
\hline Study & Study design & Participants & Study setting and database & Country & $\begin{array}{l}\text { Comorbidities in } \\
\text { associated with PU } \\
\text { development }\end{array}$ & $\begin{array}{l}\text { Comorbidities not } \\
\text { associated with PU } \\
\text { development }\end{array}$ \\
\hline $\begin{array}{l}\text { Salzberg et al, } \\
1996 \text { [6] }\end{array}$ & Case-control & 219 & $\begin{array}{l}\text { Single spinal cord injury unit in } \\
\text { veterans affair medical center }\end{array}$ & United States & $\begin{array}{l}\text { Cardiac disease, } \\
\text { diabetes mellitus, pul- } \\
\text { monary disease, renal } \\
\text { disease }\end{array}$ & $\begin{array}{l}\text { Urinary tract infec- } \\
\text { tion, sepsis }\end{array}$ \\
\hline $\begin{array}{l}\text { Cakmak et al, } \\
2009[34]\end{array}$ & Case-control & 64 & $\begin{array}{l}\text { Single physical therapy and reha- } \\
\text { bilitation hospital }\end{array}$ & Turkey & None & $\begin{array}{l}\text { Diabetes mellitus, } \\
\text { hypertension }\end{array}$ \\
\hline $\begin{array}{l}\text { Verschueren et } \\
\text { al, } 2011 \text { [35] }\end{array}$ & Cohort & 193 & $\begin{array}{l}\text { Eight rehabilitation hospitals } \\
\text { with SCI units }\end{array}$ & The Netherlands & Pulmonary disease & $\begin{array}{l}\text { Cardiovascular dis- } \\
\text { ease, spine fracture, } \\
\text { urinary tract infection }\end{array}$ \\
\hline $\begin{array}{l}\text { Wang et al, } \\
2013 \text { [36] }\end{array}$ & Case-control & 5804 & UDSMR 2009-2011 & United States & $\begin{array}{l}\text { Dementia, diabetes } \\
\text { mellitus, peripheral } \\
\text { vascular disease }\end{array}$ & $\begin{array}{l}\text { Amputation, arthritis, } \\
\text { cardiac disorders, } \\
\text { pulmonary disorders, } \\
\text { spinal cord injury, } \\
\text { stroke, }\end{array}$ \\
\hline
\end{tabular}

UDSMR (Uniform Data System for Medical Rehabilitation): A large nongovernment registry for standardized medical rehabilitation information. 
single hospital [24-29,31,33]. Some studies targeted patients who received cardiac surgery $[24,25,27]$ or hip fracture surgery[29,30]. In seven of nine cohort studies [24-26,28-32], diabetes mellitus was identified as a risk factor for PU development. This result was strongly supported by one recent meta-analysis [32] involving six observational studies with a total 2453 patients; when compared to patients with normal glucose tolerance, patients with diabetes mellitus were more likely to develop PUs (odds ratio $=2.15,95 \%$ confidential interval: 1.62-2.84). In addition, other comorbidities including cardiac disease $[27,30,33]$, renal failure [33], respiratory disease[24,30,33], and spinal cord injury [28] were also described as risk factors.

\section{Rehabilitation Setting}

Because of restrictions on mobility, patients undergoing rehabilitation are thought to be at extremely high risk for PU development. Table 4 lists four studies, including three case-control studies $[6,34,36]$ and one cohort study [35], investigating PU-related comorbidities at rehabilitation facilities [6,35-37]. Among these, three studies utilized data obtained from a single hospital $[6,34]$ or small number of hospitals [35], while one utilized a large inpatient database [36]. Two studies targeting patients admitted to spinal cord injury units [6,35]. Diabetes mellitus[6,36] and pulmonary disease $[6,35]$ were each found to be risk factors for pressure ulcer development in two studies. Additionally, cardiac disease [6], dementia [36], peripheral vascular disease[36], and renal disease [6] were also listed as risk factors.

\section{Home Setting}

Although most cases of PUs occur in acute or long-term care settings, some also occur in the home setting. The author found one cohort study [37] using a large outpatient record database that investigated the risk factors for PU development (Table 5). This study suggested the following comorbidities as risk factors: Alzheimer's disease, cerebral vascular events, chronic obstructive pulmonary disease, congestive heart failure, diabetes mellitus, deep venous thrombosis, hip fracture, limb paralysis, lower limb edema, malignancy, osteoporosis, Parkinson's disease, rheumatoid arthritis, and urinary tract infection.

\section{Discussion And Summary}

PUs can develop in various care settings. In this paper, we categorized the relevant literature published to date on the subject according to five types of care setting. Importantly, patient background, study design, sample size, and risk factor variables varied widely in each study. Moreover, a large dispersion of the results was seen between the studies, even in the same setting. For this reason the author concluded that the outcomes from each study mentioned above are in some instances not amenable to comparison. Nonetheless, among the numerous comorbidities referred to in these studies, diabetes mellitus seemed to present the strongest association, with half or more of the studies in all of the care settings and one meta-analysis pointing to this condition as a risk factor for PU development. With respect to other comorbidities,

Table 5. Home settings

\begin{tabular}{|l|l|l|l|l|l|l|}
\hline Study & Study design & Participants & $\begin{array}{l}\text { Study setting and } \\
\text { database }\end{array}$ & Country & $\begin{array}{l}\text { Comorbidities in associated with } \\
\text { PU development }\end{array}$ & $\begin{array}{l}\text { Comorbidities not } \\
\text { associated with PU } \\
\text { development }\end{array}$ \\
\hline & & & $\begin{array}{l}\text { Alzheimer's disease, diabetes mel- } \\
\text { litus, chronic heart failure, chronic } \\
\text { obstructive pulmonary disease, } \\
\text { cerebral vascular accident, deep } \\
\text { venous thrombosis, hip fracture, } \\
\text { limb paralysis, lower limb edema, } \\
\text { malignancy, osteoporosis, Parkin- } \\
\text { son's disease, rheumatoid arthritis, } \\
\text { urinary tract infection }\end{array}$ & $\begin{array}{l}\text { Angina, hypertension, } \\
\text { pneumonia }\end{array}$ \\
\hline
\end{tabular}

GPRD (General Practice Research Database): a large outpatient record database from the United Kingdom.

cardiac, renal, and respiratory disorders were commonly found to be risk factors in four of the five care settings: hospital, perioperative, rehabilitation, and home. However, the author could not find a clear difference in the kinds of PU-related comorbidities between settings.

In summary, the findings of previous studies investigating risk factors for PU development underscore the importance of recognizing patient comorbidities in order to prevent PUs. Despite the large dispersion seen between the study results, diabetes mellitus, cardiac disease, renal disease, and respiratory disease were found to be risk factors across a variety of settings.

\section{References}

[1]. European Pressure Ulcer Advisory Panel and National Pressure Ulcer Advisory Panel. Prevention and treatment of pressure ulcers: quick reference guide. National Pressure Ulcer Advisory Panel, Washington DC

[2]. Stechmiller JK, Cowan L, Whitney JD, Phillips L, Aslam R, et al. (2008) Guidelines for the prevention of pressure ulcers. Woun Repair Regen 16:
151-168.

[3]. Queensland Health. (2009) Pressure ulcer prevention and managment resource guidelines. Brisbane, Queensland Health.

[4]. The Japanese Society of Pressure Ulcers Guideline Revision Committee. (2012) JSPU Guidelines for the Prevention and Management of Pressure Ulcers.(3rd Ed) Japan J Pressure Ulcers 14: 165-226 (in Japanese).

[5]. van Marum RJ, Ooms ME, Ribbe MW, van Eijk JT. (2000) The Dutch pressure sore assessment score or the Norton scale for identifying at-risk nursing home patients? Age Ageing 29: 63-68.

[6]. Salzberg CA, Byrne DW, Cayten CG, van Niewerburgh P, Murphy JG, et al (1996) A new pressure ulcer risk assessment scale for individuals with spinal cord injury. Am J Phys Med Rehabil 75: 96-104.

[7]. Salzberg CA, Byrne DW, Kabir R, van Niewerburg P, Cayten CG. (1999) Predicting pressure ulcers during initial hospitalization for acute spinal cord injury. Wounds 11: 45-57.

[8]. Waterlow J. (1985) Pressure sores: a risk assessment card. Nurs Times 81: 49-55.

[9]. Spector WD. (1994) Correlates of pressure sores in nursing homes: evidence from the National Medical Expenditure Survey. J Invest Dermatol 102: 42S45S.

[10]. Brandeis GH, Berlowitz DR, Hossain M, Morris JN. (1995) Pressure ulcers: the Minimum Data Set and the Resident Assessment Protocol. Adv Wound Care 8: 18-25.

[11]. Brandeis GH, Ooi WL, Hossain M, Morris JN, Lipsitz LA. (1994) A longi- 
tudinal study of risk factors associated with the formation of pressure ulcers in nursing homes. J Am Geriatr Soc 42: 388-393.

[12]. Spector WD, Fortinsky RH. (1998) Pressure ulcer prevalence in Ohio nursing homes: clinical and facility correlates. J Aging Health 10: 62-80.

[13]. Berlowitz DR, Brandeis GH, Anderson JJ, Ash AS, Kader B, et al. (2001) Evaluation of a risk-adjustment model for pressure ulcer development using the Minimum Data Set. J Am Geriatr Soc 49: 872-876.

[14]. Berlowitz DR, Brandeis GH, Morris JN, Ash AS, Anderson JJ, et al. (2001) Deriving a risk-adjustment model for pressure ulcer development using the Minimum Data Set. J Am Geriatr Soc 49: 866-871.

[15]. Casimiro C, García-de-Lorenzo A, Usán L. (2002) Prevalence of decubitus ulcer and associated risk factors in an institutionalized Spanish elderly population. Nutrition. 18: 408-414.

[16]. Allman RM, Goode PS, Patrick MM, Burst N, Bartolucci AA.

[17]. (1995) Pressure ulcer risk factors among hospitalized patients with activity limitation. JAMA 273: 865-870.

[18]. Berlowitz DR, Wilking SV. (1989) Risk factors for pressure sores. A comparison of cross-sectional and cohort-derived data. J Am Geriatr Soc 37: 1043-1050.

[19]. Bianchetti A, Zanetti O, Rozzini R, Trabucchi M. (1993) Risk factors for the development of pressure sores in hospitalized elderly patients: results of a prospective study. Arch Gerontol Geriatr 16: 225-232.

[20]. Allman RM, Goode PS, Patrick MM, Burst N, Bartolucci AA. (1995) Pressure ulcer risk factors among hospitalized patients with activity limitation. JAMA 273: 865-870.

[21]. Jiricka MK, Ryan P, Carvalho MA, Bukvich J. (1995) Pressure ulcer risk factors in an ICU population. Am J Crit Care 4: 361-367.

[22]. Scott JR, Gibran NS, Engrav LH, Mack CD, Rivara FP. (2006) Incidence and characteristics of hospitalized patients with pressure ulcers: State of Washington, 1987 to 2000. Plast Reconstr Surg 117: 630-634.

[23]. Fogerty MD, Abumrad NN, Nanney L, Arbogast PG, Poulose B, et al. (2008) Risk factors for pressure ulcers in acute care hospitals. Wound Repair Regen 16: 11-18

[24]. Lyder CH, Wang Y, Metersky M, Curry M, Kliman R, et al. (2012) Hospital-acquired pressure ulcers: results from the national Medicare Patient Safety Monitoring System study. J Am Geriatr Soc 60: 1603-1608.

[25]. Papantonio CT, Wallop JM, Kolodner KB. (1994) Sacral ulcers following cardiac surgery: incidence and risks. Adv Wound Care 7: 24-36.

[26]. Lewicki LJ, Mion L, Splane KG, Samstag D, Secic M. (1997) Patient risk factors for pressure ulcers during cardiac surgery. AORN J 65: 933-942.

[27]. Schultz A, Bien M, Dumond K, Brown K, Myers A. (1999) Etiology and incidence of pressure ulcers in surgical patients. AORN J 70: 434, 437-440, 443-449.

[28]. Pokorny ME, Koldjeski D, Swanson M. (2003) Skin care intervention for patients having cardiac surgery. Am J Crit Care 12: 535-544.

[29]. Frankel H, Sperry J, Kaplan L. (2007) Risk factors for pressure ulcer development in a best practice surgical intensive care unit. Am Surg 73: 1215-1217

[30]. Haleem S, Heinert G, Parker MJ. (2008) Pressure sores and hip fractures. Injury 39: 219-223.

[31]. Lindholm C, Sterner E, Romanelli M, Pina E, Torra y Bou J, et al. (2008) Hip fracture and pressure ulcers - the Pan-European Pressure Ulcer Study intrinsic and extrinsic risk factors. Int Wound J 5: 315-328.

[32]. Slowikowski GC, Funk M. (2010) Factors associated with pressure ulcers in patients in a surgical intensive care unit. J Wound Ostomy Continence Nurs 37: 619-626.

[33]. Liu P, He W, Chen HL. (2012) Diabetes mellitus as a risk factor for surgeryrelated pressure ulcers: a meta-analysis. J Wound Ostomy Continence Nurs 39: 495-499.

[34]. O'Brien DD, Shanks AM, Talsma A, Brenner PS, Ramachandran SK. (2014) Intraoperative risk factors associated with postoperative pressure ulcers in critically ill patients: a retrospective observational study. Crit Care Med 42: 40-47.

[35]. Cakmak SK, Gül U, Ozer S, Yiğit Z, Gönü M. (2009) Risk factors for pressure ulcers. Adv Skin Wound Care 22: 412-415.

[36]. Verschueren JH, Post MW, de Groot S, van der Woude LH, van Asbeck FW, et al. (2011) Occurrence and predictors of pressure ulcers during primary inpatient spinal cord injury rehabilitation. Spinal Cord 49: 106-112.

[37]. Wang H, Niewczyk P, Divita M, Camicia M, Appelman J, et al. (2013) Impact of Pressure Ulcers on Outcomes in Inpatient Rehabilitation Facilities. Am J Phys Med Rehabil [Epub ahead of print].

[38]. Margolis DJ, Knauss J, Bilker W, Baumgarten M. (2003) Medical conditions as risk factors for pressure ulcers in an outpatient setting. Age Ageing 32: 259-264. 